\title{
Long non-coding RNA DLX6-AS1 is associated with malignant progression and promotes proliferation and invasion in esophageal squamous cell carcinoma
}

\author{
MINGCHEN WANG $^{1 *}$, YUAN LI $^{1 *}$, YIXIN YANG $^{2}$, XIAOSA LIU ${ }^{1}$, MINGXI ZANG $^{1}$, \\ YUEBAI LI ${ }^{1}, \mathrm{KUNPENG} \mathrm{YANG}^{3}$, WANGUANG YANG ${ }^{4}$ and SHANFENG ZHANG ${ }^{1}$ \\ ${ }^{1}$ Department of Biochemistry and Molecular Biology, School of Basic Medical Sciences and ${ }^{2}$ School of Clinical Medicine, \\ Zhengzhou University; ${ }^{3}$ Department of Chest Surgery, The Second Affiliated Hospital of Zhengzhou University; \\ ${ }^{4}$ Department of Emergency, The First Affiliated Hospital of Zhengzhou University, Zhengzhou, Henan 450001, P.R. China
}

Received February 2, 2018; Accepted September 10, 2018

DOI: $10.3892 / \mathrm{mmr} .2018 .9786$

\begin{abstract}
Despite being one of the most prevalent and fatal types of cancer worldwide, the biological details of esophageal squamous cell carcinoma (ESCC) remain unknown. Recent studies have demonstrated the crucial roles of long non-coding RNAs (lncRNAs) in diverse biological processes including cancer initiation, progression and metastasis. The aim of the present study was to assess the expression profile of distal-less homeobox 6 antisense RNA 1 (DLX6-AS1) in ESCC tissues and its contributions to ESCC cell proliferation, apoptosis and invasion. The expression of DLX6-AS1 in a series of ESCC samples and paired adjacent noncancerous tissues was evaluated by reverse transcription-quantitative polymerase chain reaction. Cell proliferation, apoptosis, wound healing and Transwell invasion assays were performed to evaluate the roles of DLX6-AS1 in the ESCC cell lines EC109 and KYSE30 transfected with DLX6-AS1 small interfering RNA (siRNA). Compared with the paired adjacent noncancerous tissues, DLX6-AS1 expression was upregulated in the ESCC tissues and significantly associated with differentiation status, Tumor-Node-Metastasis stage, distant metastasis, and lymph node metastasis. Knockdown of DLX6-AS1 significantly suppressed cell proliferation, invasion and migration abilities, and enhanced the apoptotic rate in the two ESCC cell lines. Furthermore, western blot assays revealed that silencing DLX6-AS1 partly influenced the epithelial-mesenchymal transition process in ESCC cells. These
\end{abstract}

Correspondence to: Mr. Shanfeng Zhang, Department of Biochemistry and Molecular Biology, School of Basic Medical Sciences, Zhengzhou University, 100 Kexue Avenue, Zhengzhou, Henan 450001, P.R. China

E-mail: zsf@zzu.edu.cn

*Contributed equally

Key words: long non-coding RNA, distal-less homeobox 6 antisense RNA 1, epithelial-mesenchymal transition, esophageal squamous cell carcinoma results imply that the oncogenic function of DLX6-AS1 may be a novel candidate target for treating human ESCC.

\section{Introduction}

Esophageal cancer (EC) is considered to be one of the most invasive carcinomas and is ranked sixth among the lethal aggressive carcinomas globally. EC is considered to be a fatal disease for patients diagnosed during the advanced stage, with an overall survival rate of $10-20 \%$ (1). The incidence and mortality rates associated with EC continue to rise, displaying great geographical and sociocultural variation as well as a tendency for familial aggregation in recent years, as is evident in the central regions of China (2). The main histological types of EC include adenocarcinoma and squamous cell carcinoma based on etiology and pathological features (3). Esophageal squamous cell carcinoma (ESCC) is the most prevalent histological subclass that is derived from esophageal epithelial cells (4). Although advancements in surgery, chemotherapy and radiotherapy have been achieved to treat ESCC patients, the overall 5-year survival rate still remains $<40 \%$ worldwide owing to the high incidence of local invasion and distant metastasis, particularly in late-stage ESCC patients (5). The prognosis of patients with ESCC remains poor as the detailed mechanisms of the molecular and genetic alterations underlying ESCC progression are unknown. Therefore, investigating novel biomarkers, therapeutic agents and mechanisms underlying ESCC initiation and progression are pivotal in improving the early diagnosis and treatment of ESCC.

Long non-coding RNAs (IncRNAs) are a novel transcript class of RNA molecules, longer than 200 nucleotides, which lack the capacity to be translated into proteins (6). LncRNAs are aberrantly expressed or dysregulated in a variety of physiological and pathological processes via complex mechanisms $(3,7)$. Accumulating evidence has confirmed that IncRNAs essentially regulate the expression of their downstream-reacting genes at the epigenetic, transcriptional and post-transcriptional levels (8). LncRNAs have been associated with the genesis, progression and prognosis of various types of cancer, and increasingly emerge either as tumor suppressors 
or promoters that potentially possess oncogenic functions (9). Furthermore, lncRNAs perform their respective biological functions at the RNA level and also regulate microRNA (miR) function by competing with target mRNAs (10). Although a decade of research has contributed to establishing the mechanism underlying the function of lncRNAs, a large number of IncRNAs with potential roles, particularly in cancer, have not been discovered or thoroughly investigated. In the past few years, previous studies have indicated a close association between the abnormal expression of lncRNAs and several malignant types of cancer including human EC, suggesting that IncRNAs could serve important roles in the initiation of $\mathrm{EC}$, with particular emphasis on $\operatorname{ESCC}(7,11,12)$.

The lncRNA, distal-less homeobox 6 antisense 1 (DLX6-AS1), is localized at the 7q21.3 chromosomal region in humans and is transcribed as a 1,990 bp noncoding RNA. Previous studies have suggested that DLX6-AS1 may function as a noncoding oncogene, and that its dysregulation is closely associated with the progression and poor prognosis of tumors in lung adenocarcinoma (13), renal cell carcinoma (14) and hepatocellular carcinoma (15). These studies implied that tumorigenesis or progression was associated with the dysregulation of DLX6-AS1. However, the potential mechanisms underlying the role of DLX6-AS1 in ESCC have yet to be elucidated and the associated studies are few in number. In the present study, the expression profile of DLX6-AS1 was characterized in ESCC tissues and the adjacent histologically normal tissues. The association between the expression patterns of DLX6-AS1 and the clinical characteristics of patients with ESCC were also investigated. Furthermore, the functional impact of DLX6-AS1 on ESCC cell proliferation, apoptosis and invasive ability was investigated by performing in vitro lncRNA knockdown assays. The results revealed the potential roles of DLX6-AS1 and provided a novel perspective on the progression and pathogenesis of ESCC.

\section{Materials and methods}

Clinical tissue samples. Paired primary ESCC and matched adjacent non-neoplastic specimens were obtained from 73 eligible patients enrolled in the present study at the First Affiliated Hospital of Zhengzhou University (Zhengzhou, China) between April 2015 and July 2016 (Table I). All of the biopsy samples were obtained from ESCC patients who did not undergo chemotherapy or radiotherapy and were not suffering from any other serious diseases. Following surgical resection, the samples were frozen and stored using liquid nitrogen until RNA extraction. The histopathology of the tumor tissues was independently reviewed by at least two pathologists. The Union for International Cancer Control/American Joint Committee on Cancer classification guidelines were applied to determine the tumor stage in each individual (16). The acquired clinicopathological information for all of the samples is available in Table I. Written informed consent was obtained from each patient prior to using the clinical samples for research purposes. The Ethics Committee of Zhengzhou University (Henan, China) approved the study protocol.

Cell culture. The human ESCC cell lines, EC109 and KYSE30, were purchased from the Shanghai Institute of Biochemistry and Cell Biology (Chinese Academy of Sciences, Shanghai, China). The human immortalized normal esophageal epithelial cell line, Het-1A, was obtained from American Type Culture Collection (ATCC, Manassas, VA, USA). All of the cells were maintained in RPMI-1640 medium (Gibco; Thermo Fisher Scientific, Inc., Waltham, MA, USA) supplemented with $10 \%$ fetal bovine serum (Gibco; Thermo Fisher Scientific, Inc.), $100 \mathrm{U} / \mathrm{ml}$ penicillin and $100 \mu \mathrm{g} / \mathrm{ml}$ streptomycin at $37^{\circ} \mathrm{C}$ in a humidified chamber containing $5 \% \mathrm{CO}_{2}$.

Cell transfection. Small interfering RNAs (siRNAs) specifically targeting 1ncRNA DLX6-AS1 were synthesized by Shanghai GenePharma Co., Ltd. (Shanghai, China) including a negative control siRNA with no definite target. EC109 and KYSE30 cells of $5 \times 10^{4}$ cells $/ \mathrm{ml}$ were cultured overnight in 6-well plates till $70 \%$ confluency was reached and then transfected with $10 \mathrm{nmol} / 1$ lncRNA DLX6-AS1 siRNA (si-lnc) or the negative control (si-NC) using Lipofectamine $2000^{\mathrm{TM}}$ (Invitrogen; Thermo Fisher Scientific, Inc.), according to the manufacturer's protocol. The cells were collected following $48 \mathrm{~h}$ of transfection to determine the efficiency of the knockdown via reverse transcription-quantitative polymerase chain reaction (RT-qPCR) analysis and other functional experiments. The target sequence for the lncRNA DLX6-AS1 siRNA was as follows: 5'-AAUAAAGAACACUUACACUACUG-3' (15).

Cell proliferation assay. To analyze the growth of the EC109 and KYSE30 cell lines, a Cell Counting Kit-8 (CCK-8; Beyotime Institute of Biotechnology, Haimen, China) assay was performed according to the manufacturer's protocol. Log-phase cells were harvested and cultured at a density of $5 \times 10^{3}$ cells/well in a 96-well plate, with quintuplicate wells per group. Following transfection with either lncRNA DLX6-AS1 siRNA or si-NC for 24,48 or $72 \mathrm{~h}$, the cells were treated with the CCK-8 solution $(10 \mu \mathrm{l})$ in the plates and incubated for $2 \mathrm{~h}$ at $37^{\circ} \mathrm{C}$. The optical density was measured for each well at a wavelength of $450 \mathrm{~nm}$ using a microplate reader (Infinite M200; Tecan Group, Ltd., Männedorf, Switzerland) to estimate cell viability. Growth curves were plotted using the mean results obtained independently from triplicate experiments.

Apoptosis analysis. The Annexin V-fluorescein isothiocyanate (FITC)/propidium iodide (PI) Apoptosis Detection kit (Beyotime Institute of Biotechnology) was used to quantify the apoptotic rate of the ESCC cells. Briefly, cells were harvested from each group via $0.25 \%$ trypsinization following a $48 \mathrm{~h}$ transfection. The cells were washed with PBS, resuspended in Annexin-binding buffer and adjusted to a density of $1 \times 10^{6}$ cells $/ \mathrm{ml}$. They were then vortexed and analyzed using a flow cytometer (BD Biosciences, Franklin Lakes, NJ, USA) following incubation with a working solution of FITC-Annexin V and PI at room temperature for $15 \mathrm{~min}$. The early and late apoptotic cells were counted and distinguished from the viable and dead cells using FlowJo software (version 7.6, FlowJo LLC, Ashland, OR, USA). Relative apoptotic ratios were calculated and compared with those of the control group. Each assay was performed independently in triplicate.

Invasion assay. The Matrigel-coated Transwell chamber system (8- $\mu \mathrm{m}$ pore size; Corning Incorporated, Corning, NY, 
Table I. Association between the clinicopathological factors and the expression of DLX6-AS1.

Expression of DLX6-AS1

\begin{tabular}{|c|c|c|c|}
\hline Characteristics & Low-DLX6-AS1 group (n) & High-DLX6-AS1 group (n) & P-value \\
\hline Sex & & & 0.393 \\
\hline Male & 23 & 20 & \\
\hline Female & 13 & 17 & \\
\hline Age, years & & & 0.549 \\
\hline$<60$ & 21 & 19 & \\
\hline$\geq 60$ & 15 & 18 & \\
\hline Tumor location & & & 0.693 \\
\hline Upper 1/3 & 9 & 8 & \\
\hline Middle 1/3 & 14 & 12 & \\
\hline Lower $1 / 3$ & 13 & 17 & \\
\hline Histological grade & & & 0.004 \\
\hline Low differentiation & 8 & 19 & \\
\hline Middle differentiation & 10 & 12 & \\
\hline High differentiation & 18 & 6 & \\
\hline Lymph node metastasis & & & 0.003 \\
\hline Yes & 14 & 27 & \\
\hline No & 22 & 10 & \\
\hline TNM stage & & & 0.024 \\
\hline $\mathrm{I} / \mathrm{II}$ & 25 & 16 & \\
\hline III/IV & 11 & 21 & \\
\hline
\end{tabular}

DLX6-AS1, distal-less homeobox 6 antisense 1; TNM, Tumor-Node-Metastasis.

USA) was used to analyze the invasive capabilities of EC109 and KYSE30 cells as per the manufacturer's protocol. The cell suspension $(200 \mu \mathrm{l})$, at a concentration of $1 \times 10^{5}$ cells $/ \mathrm{ml}$, containing serum-free RPMI-1640 medium following $48 \mathrm{~h}$ of transfection, was loaded in the upper chamber containing a membrane coated with Matrigel. Additionally, $500 \mu$ l culture medium supplemented with $10 \%$ FBS was added to the lower chamber as the chemoattractant. The system was then incubated for $48 \mathrm{~h}$ at $37^{\circ} \mathrm{C}$ and $5 \% \mathrm{CO}_{2}$. Following incubation, the noninvasive cells on the surface of the upper membrane were scraped off using a cotton swab. The cells that successfully invaded through the filter to the lower regions of the chambers were fixed with $100 \%$ methanol for $15 \mathrm{~min}$ at $4^{\circ} \mathrm{C}$, stained with $0.1 \%$ crystal violet for $15 \mathrm{~min}$ at $37^{\circ} \mathrm{C}$ and dried for $30 \mathrm{~min}$ at $80^{\circ} \mathrm{C}$. A total of five randomly selected fields per chamber were captured using a bright-field microscope (magnification, $\mathrm{x} 200$ ) to evaluate the invasive ability of the ESCC cells. Data was obtained from three independent experiments.

Cell migration assay. EC109 and KYSE30 cell motility was assessed using a scratch wound healing assay by measuring the movement of cells in an artificially scraped wound. The cells at concentration of $5 \times 10^{4}$ cells $/ \mathrm{ml}$ were seeded onto plastic 6-well plates until cell confluence reached $\sim 80 \%$ following $24 \mathrm{~h}$ of transfection. A $200 \mu \mathrm{l}$ pipette tip was used to scratch and create wounds in the confluent monolayer cell cultures, followed by 3 washes with PBS to remove the debris and floating cells. Images of wound closure were captured following 0 and $48 \mathrm{~h}$ of incubation using a Leica DMIL inverted microscope to assess the level of migration in each group of transfected cells. A total of 5 random fields per well were captured to quantify migration by measuring the distance that the cells had migrated toward the original wound field using Image Pro Plus v6.0 software (Media Cybernetics Inc., Rockville, MD, USA). Each experiment was performed independently in triplicate.

RNA isolation and RT-qPCR analysis. The RT-qPCR assays were conducted to detect the expression of DLX6-AS1. TRIzol reagent (Invitrogen; Thermo Fisher Scientific, Inc.) was used to extract total RNA from ESCC tissues or cells stored at $-80^{\circ} \mathrm{C}$, according to the manufacturer's protocol. The cDNAs for subsequent qPCRs were synthesized using the PrimeScript RT reagent kit (Takara Biotechnology Co., Ltd., Dalian, China) by reverse transcribing $1 \mu \mathrm{g}$ of total RNA using random primers at $42^{\circ} \mathrm{C}$ for $30 \mathrm{~min}$ and $85^{\circ} \mathrm{C}$ for $5 \mathrm{~min}$. PCR amplification was conducted using the Applied Biosystems 7500 fast real-time PCR system (Thermo Fisher Scientific, Inc.) in $20 \mu \mathrm{l}$ reaction mixtures containing SYBR Green Real-time PCR Master Mix (Takara Biotechnology Co., Ltd.) at $95^{\circ} \mathrm{C}$ for $30 \mathrm{sec}$ of initial denaturation, followed by 40 cycles of $95^{\circ} \mathrm{C}$ for $5 \mathrm{sec}$ and $60^{\circ} \mathrm{C}$ for $30 \mathrm{sec}$. The $\mathrm{Cq}$ value was used to determine the relative expression levels of DLX6-AS1 and to calculate the fold-change; the expression levels were internally normalized 
to that of $\beta$-actin using the $2^{-\Delta \Delta \mathrm{Cq}}$ method (17). The primers used for analysis are as follows: lncRNA DLX6-AS1 forward, 5'-AGTTTCTCTCTAGATTGCCTT-3' and reverse, 5'-ATT GACATGTTAGTGCCCTT-3'; $\beta$-actin forward, 5'-TCC CTGGAGAAGAGCTACGA-3' and reverse, 5'-ACCTGA GGCTTTGGATTCCT-3'. Each experiment was performed independently in triplicate.

Western blot analysis. EC109 and KYSE30 cells in each group were lysed using a radioimmunoprecipitation assay buffer (Beyotime Institute of Biotechnology) containing the protease inhibitor phenylmethylsulfonyl fluoride (Beyotime Institute of Biotechnology), as per the manufacturer's protocol for total protein extraction. The protein concentration was determined via a bicinchoninic acid assay (Thermo Fisher Scientific, Inc.). Protein samples $(50 \mu \mathrm{g})$ were separated using 10\% SDS-PAGE and then transferred to a nitrocellulose membrane (EMD Millipore, Billerica, MA, USA). Following blocking with $5 \%$ non-fat milk for $2 \mathrm{~h}$ at $4^{\circ} \mathrm{C}$, the membrane was subsequently incubated overnight at $4^{\circ} \mathrm{C}$ with primary antibodies in Tris-buffered saline (TBS). Primary antibodies against epithelial (E)-cadherin (1:800; ab76055; Abcam, Cambridge, UK), neural (N)-cadherin (1:800; ab98952; Abcam), vimentin (1:800; 49636; Cell Signaling Technology, Inc., Danvers, MA, USA) and $\beta$-actin (1:1,000; ab6276; Abcam) were used for western blot analysis. Following washing with TBS containing $0.1 \%$ Tween-20, the membrane was incubated with secondary antibodies (horseradish peroxidase-conjugated goat anti-mouse Immunoglobulin G; 1:2,000; HAF007; R\&D Systems, Inc., Minneapolis, MN, USA) for $2 \mathrm{~h}$ at $37^{\circ} \mathrm{C}$. Normalized to the $\beta$-actin protein density, the target protein band densities were measured using an enhanced chemiluminescence array (Beyotime Institute of Biotechnology) and densitometry were detected by Image-Pro Plus software version 6.0 (Media Cybernetics, Inc., Rockville, MD, USA).

Statistical analysis. Statistical analysis was conducted using SPSS software (version 21.0; IBM, Corps., Armonk, NY, USA). All of the data is presented as the mean \pm standard deviation. The statistical differences were evaluated using independent two-tailed Student's t-tests, one-way analysis of variance and Dunnett's test, chi-square tests. $\mathrm{P}<0.05$ was considered to indicate a statistically significant difference.

\section{Results}

Expression profile of DLX6-AS1 in human ESCC tissues and cell lines. To identify the biological roles of DLX6-AS1 in ESCC development, RT-qPCR analysis was conducted to assess the expression of DLX6-AS1 in tumor tissues $(n=73)$ and paired adjacent normal tissues $(n=73)$. As illustrated in Fig. 1A,DLX6-AS1 expression was significantly elevated in the ESCC tissue samples when compared with the corresponding adjacent noncancerous tissue $(\mathrm{P}<0.01)$. The significantly increased expression of DLX6-AS1 in advanced tumor stages (III/IV) compared with early-stage tumors (I/II) suggested that the overexpression of DLX6-AS1 may be associated with the development of ESCC ( $\mathrm{P}<0.01$; Fig. 1B). Furthermore, the levels of DLX6-AS1 were validated in the ESCC cell lines (EC109 and KYSE30) via RT-qPCR normalized to Het-1A.
The expression level of DLX6-AS1 was significantly upregulated in the two ESCC cell lines when compared with the Het-1A cell line $(\mathrm{P}<0.01$; Fig. $1 \mathrm{C})$. It was also demonstrated that the KYSE30 cells exhibited increased expression of DLX6-AS1 when compared with EC109 cells. Based on these results, the EC109 and KYSE30 cell lines were used for the subsequent experiments to evaluate the effect of DLX6-AS1 in ESCC pathogenesis.

Overexpression of DLX6-AS1 is associated with the clinicopathological characteristics of ESCC patients. To further determine whether the expression profile of DLX6-AS1 was associated with the clinicopathological parameters of ESCC, using the DLX6-AS1 expression of adjacent noncancerous tissues as a reference, the 73 patients were divided into DLX6-AS1 low- and high-expression groups, taking the median expression level of DLX6-AS1 as a cutoff (Table I). The analysis demonstrated that increased DLX6-AS1 expression was mainly detected in late-stage ESCC tissues and was significantly associated with differentiation status $(\mathrm{P}=0.004)$, lymph node metastasis $(\mathrm{P}=0.003)$ and advanced Tumor-Node-Metastasis (TNM) stage $(\mathrm{P}=0.024)$. There was insufficient evidence significantly associating the expression of DLX6-AS1 with other clinical characteristics including sex, age and tumor location. Significant associations between the aberrant expression patterns of DLX6-AS1 and the clinicopathological characteristics of ESCC patients indicated that further functional studies should be conducted in order to evaluate the role of DLX6-AS1 in the progression of ESCC.

Knockdown of DLX6-AS1 inhibits the proliferation of ESCC cells. To further understand the biological roles of DLX6-AS1 in ESCC cell phenotypes, knockdown assays were performed by transfecting EC109 and KYSE30 cell lines with the DLX6-AS1 siRNA as these cell lines contain high levels of DLX6-AS1. The success of the $48 \mathrm{~h}$ siRNA transfection was verified via RT-qPCR in EC109 and KYSE30 cells. As demonstrated in Fig. 2A and B, the expression levels of DLX6-AS1 did not differ significantly between the non-transfected group (Blank) and the nonsense siRNA transfected group (NC), suggesting that the nonsense siRNA had no effect on the expression levels of DLX6-AS1 in the ESCC cells. The expression profile of DLX6-AS1 was significantly reduced in the EC109 and KYSE30 cells following transfection with the IncRNA DLX6-AS1 siRNA (si-lnc group) compared with the Blank and NC groups $(\mathrm{P}<0.01)$. Next, a CCK- 8 assay was performed to further determine the cell viabilities of the EC109 and KYSE30 cells. As demonstrated in Fig. 2C and D, the corresponding EC109 and KYSE30 cell proliferation curves indicated that the cell viabilities of the si-lnc group were significantly inhibited at 24, 48 and $72 \mathrm{~h}$ when compared with the Blank and NC groups $(\mathrm{P}<0.05)$. Furthermore, no significant differences in cell growth were detected between the Blank and NC groups. The results of the present study suggested that suppressing DLX6-AS1 expression impeded the growth and survival capacity of EC109 and KYSE30 cells in vitro and that DLX6-AS1 promoted ESCC cell proliferation.

Silencing DLX6-AS1 promotes the apoptosis of EC109 and KYSE30 cells. To investigate the potential mechanisms 

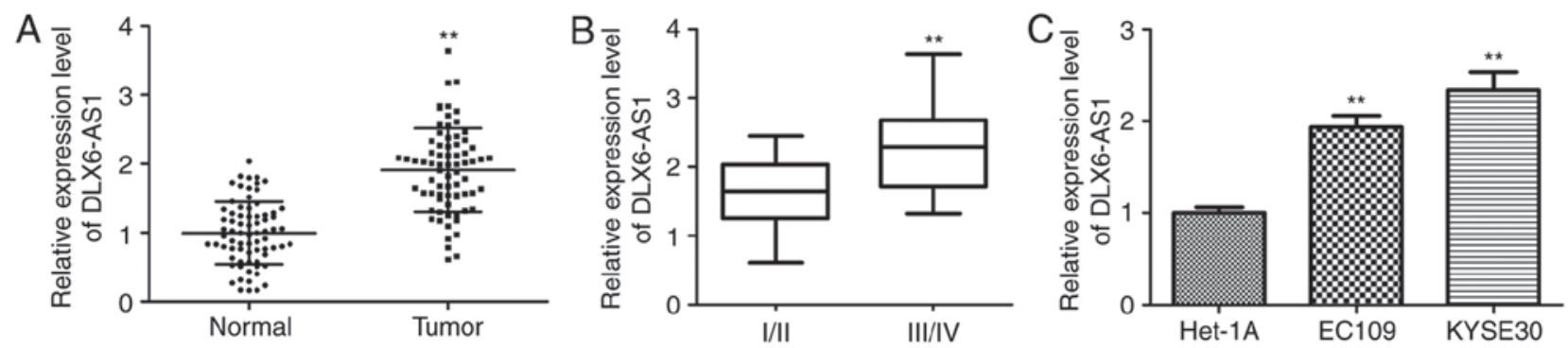

Figure 1. Expression profile of DLX6-AS1 in human ESCC tissues and cell lines. (A) The expression levels of DLX6-AS1 in human ESCC tissues and the corresponding adjacent normal tissues compared with $\beta$-actin were detected by RT-qPCR ( $n=73)$. ${ }^{* * *} \mathrm{P}<0.01$ compared with the normal group. (B) Expression of DLX6-AS1 was significantly higher in patients with an advanced Tumor-Node-Metastasis stage. ${ }^{* *} \mathrm{P}<0.01$ compared with the I/II group. (C) RT-qPCR determined the expression levels of DLX6-AS1 in two ESCC cell lines (EC109 and KYSE30) and a normal esophageal cell line (Het-1A) compared with $\beta$-actin. ${ }^{* *} \mathrm{P}<0.01$ compared with the Het-1A cells. Data is presented as the mean \pm standard deviation. ESCC, esophageal squamous cell carcinoma; DLX6-AS1, distal-less homeobox 6 antisense RNA 1; RT-qPCR, reverse transcription-quantitative polymerase chain reaction.
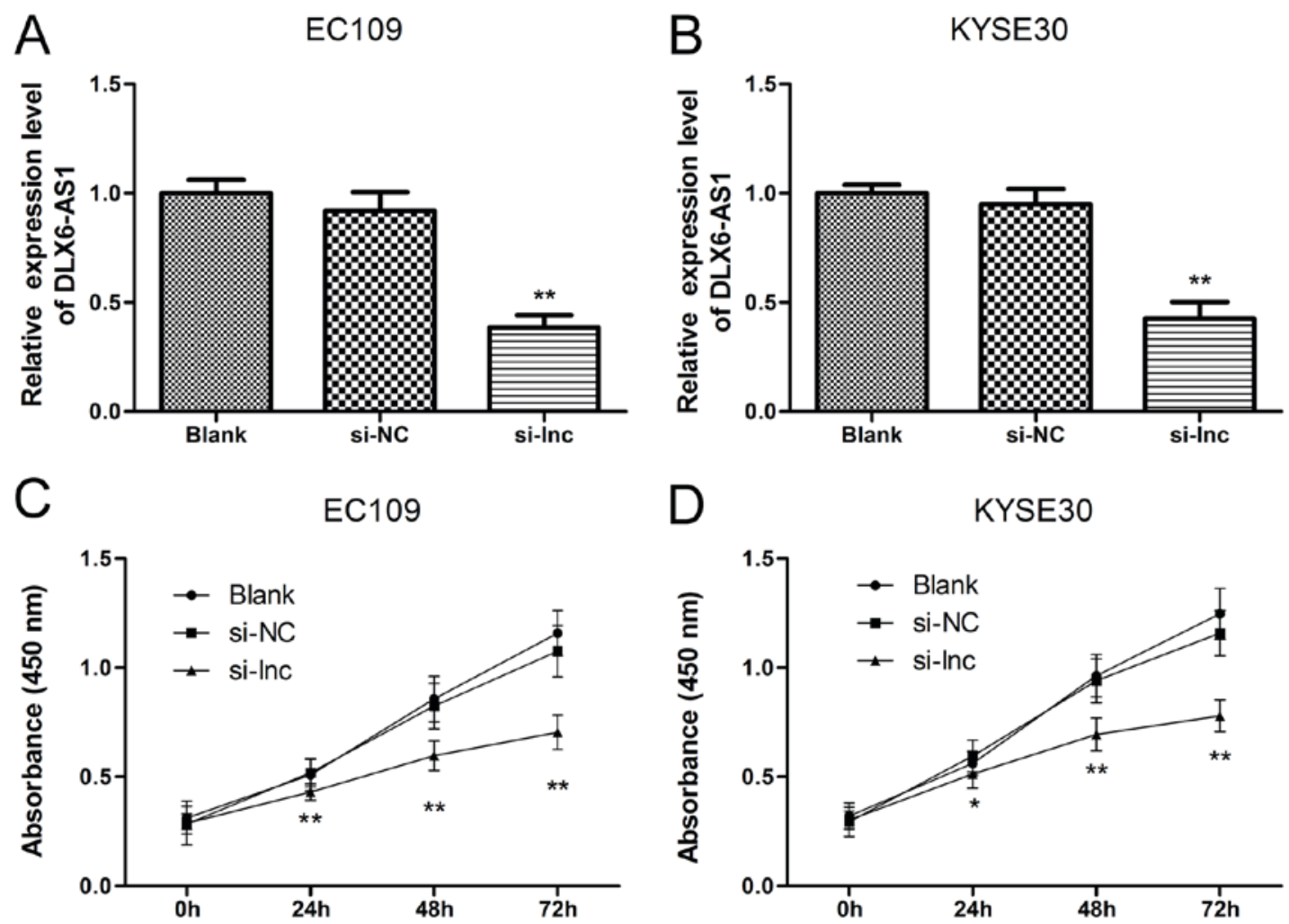

Figure 2. Knockdown of DLX6-AS1 inhibits the proliferation of ESCC cells. The results of DLX6-AS14 expression in the ESCC cell lines (A) EC109 and (B) KYSE30 transfected with si-lnc, si-NC, or Blank were validated by reverse transcription-quantitative polymerase chain reaction. ${ }^{* * *} \mathrm{P}<0.01$ compared with the si-NC groups. The expression of DLX6-AS14 was significantly downregulated in the si-lnc group. Cell Counting Kit-8 assays demonstrated a significant reduction in cell proliferation in (C) EC109 and (D) KYSE30 cells transfected with DLX6-AS1 siRNA in the si-lnc group. ${ }^{*} \mathrm{P}<0.05$ and ${ }^{* * *} \mathrm{P}<0.01$ compared with the si-NC groups. Data are presented as the mean \pm standard deviation. si-NC, nonsense small interfering RNA negative control; si-lnc, DLX6-AS1 siRNA; ESCC, esophageal squamous cell carcinoma; DLX6-AS1, distal-less homeobox 6 antisense RNA 1; Blank, non-transfected.

underlying the inhibition of cell proliferation induced by DLX6-AS1 knockdown in ESCC cells, flow cytometry assays were performed to examine the apoptosis process in EC109 and KYSE30 cells transfected with lncRNA DLX6-AS1 siRNA for $48 \mathrm{~h}$. As illustrated in Fig. 3, the percentage of apoptotic EC109 and KYSE30 cells significantly increased following DLX6-AS1 knockdown compared with the Blank and NC groups $(\mathrm{P}<0.01)$. Consistent with cell proliferation assays, these flow cytometry results demonstrated that silencing DLX6-AS1 using siRNA induced apoptosis in EC109 and KYSE30 cells.
Downregulation of DLX6-AS1 inhibits invasion and motility of EC109 and KYSE30 cells. Transwell and wound healing assays were conducted to investigate the roles of DLX6-AS1 in the invasion and motility of the ESCC cell lines. The Transwell assay results demonstrated that the number of cells penetrating the Transwell membrane containing Matrigel did not differ significantly between the Blank and NC groups. However, significantly fewer invasive cells were observed in the si-lnc group when compared with the Blank and NC groups $(\mathrm{P}<0.01$; Fig. $4 \mathrm{~A})$. This was further confirmed in the wound healing assays, which 

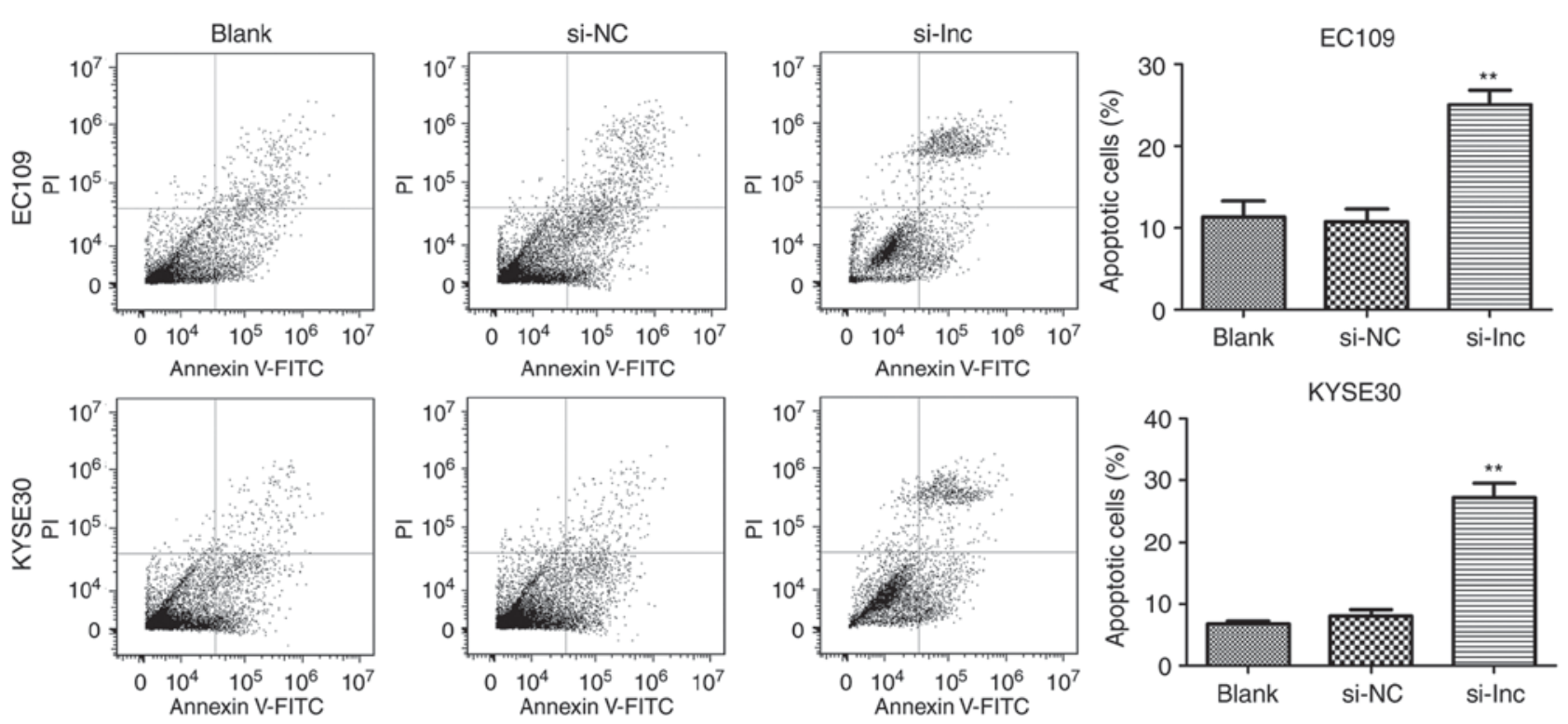

Figure 3. Silencing distal-less homeobox 6 antisense RNA 1 promotes the apoptosis of EC109 and KYSE30 cells. EC109 and KYSE30 cells transfected with si-lnc demonstrated a significantly increased response to apoptosis. ${ }^{* *} \mathrm{P}<0.01$ compared with the si-NC groups. Data are presented as the mean \pm standard deviation. si-NC, nonsense small interfering RNA negative control; si-lnc, distal-less homeobox 6 antisense RNA 1; siRNA; FITC, fluorescein isothiocyanate; PI, propidium iodide; Blank, non-transfected.

demonstrated that the wound closure was significantly inhibited in the EC109 and KYSE30 cells transfected with 1ncRNA DLX6-AS1 siRNA when compared with the Blank and NC groups ( $\mathrm{P}<0.01$; Fig. 4B). Collectively, the results indicated that the invasive capabilities and motilities of EC109 and KYSE30 cells were significantly suppressed by the lncRNA DLX6-AS1 siRNA treatment when compared with the Blank and NC groups $(\mathrm{P}<0.01$; Fig. 4). Therefore, DLX6-AS1 is involved in mechanisms that promote ESCC cell invasion and motility.

Effect of DLX6-AS1 expression on the epithelial-mesenchymal transition (EMT) of ESCC cells. To investigate the mechanisms underlying the knockdown of DLX6-AS1 that inhibits the invasion and migration of ESCC cells, the expression of EMT markers was analyzed as a result of their involvement in cancer metastatic potential and progression. The present study sought to determine if the abnormal expression of DLX6-AS1 was associated with epithelial and mesenchymal features of the ESCC cell phenotype. The expression of the EMT-associated markers was measured in the EC109 and KYSE30 cells via western blotting assays following transfection with DLX6-AS1 siRNA. As demonstrated in Fig. 5, DLX6-AS1 knockdown in EC109 and KYSE30 cells resulted in significant overexpression of E-cadherin (epithelial marker), and significantly reduced the expressions of $\mathrm{N}$-cadherin and vimentin (mesenchymal markers) when compared with the expression levels of these proteins in the Blank and NC groups $(\mathrm{P}<0.01)$. Therefore, the results implied that DLX6-AS1 suppression markedly inhibited ESCC cell metastasis by affecting the EMT process.

\section{Discussion}

Genome-wide analysis has demonstrated that non-protein coding RNAs occupy $>90 \%$ of the human genome's transcriptional output in genome-wide analysis (18); in addition, the detailed molecular mechanisms for the majority of lncRNAs have yet to be elucidated. Accumulating evidence has illustrated that lncRNAs serve essential roles during gene regulatory processes and are involved in a number of biological functions, including cell proliferation and differentiation in normal and transformed cells $(19,20)$. Emerging evidence has further demonstrated that dysregulation of lncRNAs is involved in epigenetic regulatory mechanisms underlying physiological and pathological processes; therefore, lncRNAs serve as novel regulators in progressive and uncontrolled tumor growth, EMT processes and metastasis $(12,21,22)$. It is essential to investigate tumor-associated lncRNAs in order to recognize their complex intermolecular interactions in carcinogenesis, specifically esophageal cancer. Rapid growth and high incidence of regional and distant metastasis of aggressive tumors contributes to the poor prognosis of ESCC patients, despite rapid advances in the diagnosis and treatment of the disease (23). A clear understanding of the critical mechanisms involved in the genesis and development of ESCC is required in order to improve the survival rates of ESCC patients. Certain investigators have demonstrated that the abnormal expression of certain lncRNAs, including lncRNAs HOX transcript antisense RNA (19), POU class 3 homeobox 3 (24) and SPRY4-intronic transcript 1 (11), is associated with the progression of ESCC. Therefore, focusing on specific lncRNAs, which function clinically as tumor promoters or suppressors during the progression of ESCC with biomarker applications, can help to improve the diagnosis and treatment of ESCC. The potential role of DLX6-AS1 in the clinical prognostic prediction of ESCC focuses attention on its underlying mechanisms in ESCC.

Recent studies have illustrated that DLX6-AS1 can act as a positive prognostic marker for the diagnosis of various types of cancer, including lung adenocarcinoma, hepatocellular carcinoma and renal cell carcinoma (13-15). The overexpression 
A
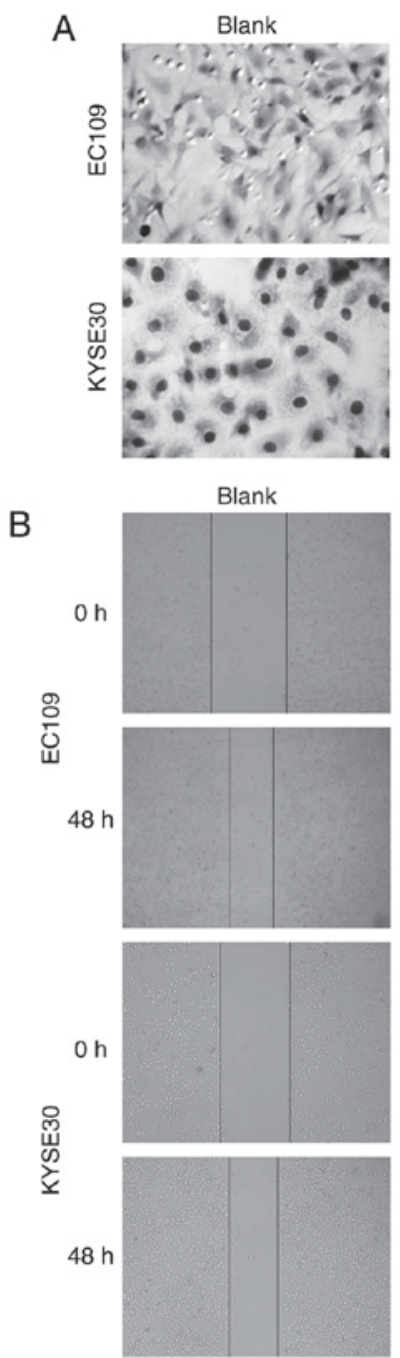

si-NC

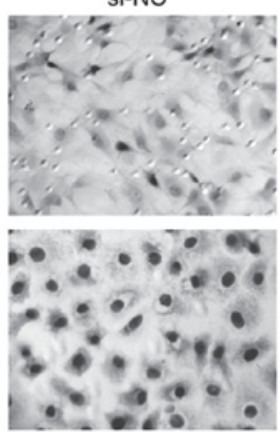

si-NC
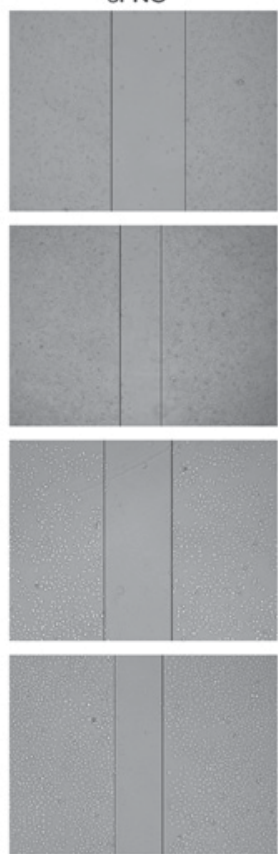

si-Inc

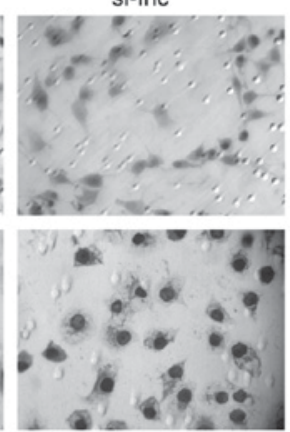

si-Inc
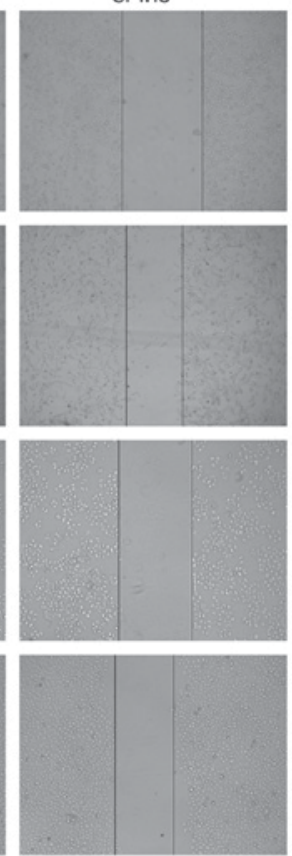

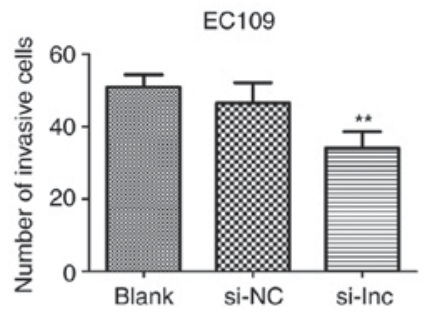

KYSE30

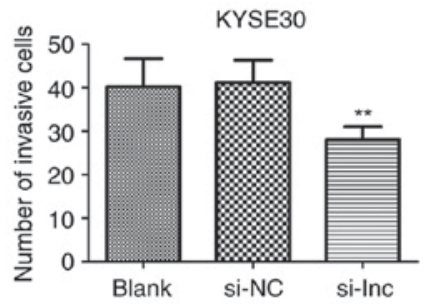

EC109
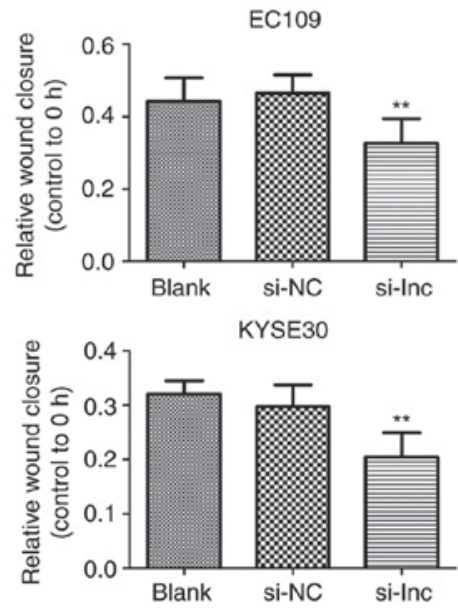

Figure 4. Downregulation of DLX6-AS1 inhibits the invasion and motility of EC109 and KYSE30 cells. (A) Transwell assays (magnification, x200) demonstrated the differences in the invasive ability of EC109 and KYSE30 cells transfected with si-lnc, si-NC, or Blank at 0 and 48 h, respectively. The invasive ability of EC109 and KYSE30 cells was significantly suppressed in the si-lnc groups. ${ }^{* *} \mathrm{P}<0.01$ compared with the si-NC groups. (B) The wound healing assays (magnification, x50) demonstrated that the migratory ability of EC109 and KYSE30 cells was significantly inhibited in the si-lnc groups. ${ }^{* *}$ P $<0.01$ compared with the si-NC groups. Data is presented as the mean \pm standard deviation. si-NC, nonsense small interfering RNA negative control; si-lnc, DLX6-AS1 siRNA; DLX6-AS1, distal-less homeobox 6 antisense RNA 1; blank, non-transfected.

of DLX6-AS1 contributes to the metastasis and invasion of lung adenocarcinoma cells and is positively associated with the carcinogenesis, advanced histological differentiation and TNM stage of lung adenocarcinoma (13). Abundance of DLX6-AS1 also promoted the growth, invasion and migration of hepatocellular carcinoma cells in in vivo and in vitro assays and effectively predicted the poor prognosis of hepatocellular carcinoma patients (15). Its expression is also elevated in renal cell carcinoma samples and associated with the progression and metastasis of renal cell carcinoma, indicating that DLX6-AS1 be an oncogenic agent in renal cell carcinoma (14). However, the underlying mechanism and role of DLX6-AS1 in ESCC remains uninvestigated. Based on these associated studies, preliminary functional experiments were performed to investigate the expression levels and working mechanisms of DLX6-AS1 in ESCC.

The results of the present study confirmed that the expression of DLX6-AS1 significantly increased in the majority of
ESCC tissues and cell lines when compared with the adjacent normal tissue. Aberrant DLX6-AS1 expression was also positively associated with the clinical prognostic factors of ESCC. To further understand the roles of DLX6-AS1 in ESCC metastasis and progression, loss-of-function assays were conducted to suppress the expression of DLX6-AS1 in EC109 and KYSE30 cells with the help of specifically designed siRNA. Knockdown of DLX6-AS1 resulted in the inhibition of the proliferative, migratory and invasive capabilities of the ESCC cell lines, and induced apoptosis in vitro in the two ESCC cell lines compared with the control. Previous studies have demonstrated that cancer cells become motile and invasive via the EMT process by losing the epithelial phenotype and acquiring mesenchymal properties, which is associated with invasion and metastasis in many types of cancer, including ESCC $(25,26)$. EMT, in particular, has been associated with metastasis and mortality in ESCC patients, and is regulated by several lncRNAs $(11,22,27)$. However, DLX6-AS1's function 
A
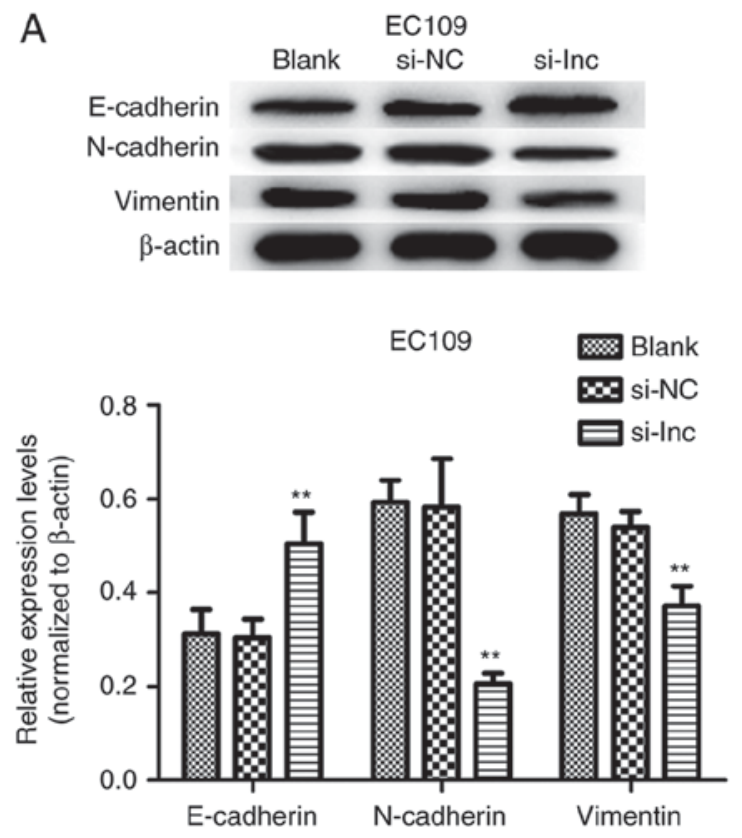

B
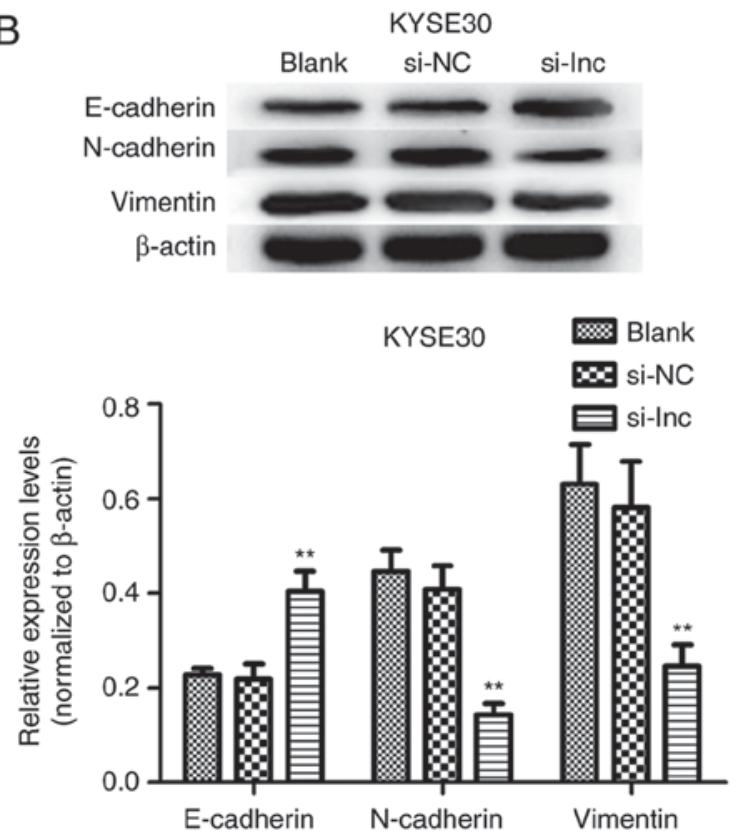

Figure 5. Effect of the expression of DLX6-AS1 on the regulation of EMT in ESCC. The expression levels EMT markers (E-cadherin, N-cadherin, and Vimentin) in (A) EC109 and (B) KYSE30 cells transfected with si-lnc, si-NC or Blank at 48 h were analyzed by western blotting. Knockdown of DLX6-AS1 significantly enhanced the expression of E-cadherin, and suppressed the expression of N-cadherin and Vimentin in EC109 and KYSE30 cells in the si-lnc groups. ${ }^{* *} \mathrm{P}<0.01$ compared with the si-NC groups. $\beta$-Actin was used as a loading control. Data are presented as the mean \pm standard deviation. si-NC, nonsense small interfering RNA negative control; si-lnc, DLX6-AS1 siRNA; DLX6-AS1, distal-less homeobox 6 antisense RNA 1; E, epithelial; N, neural; EMT, epithelial-mesenchymal transition; Blank, non-transfected.

in EMT has not been demonstrated until now. In the present study, the abnormal expression of DLX6-AS1 regulated the expression levels of EMT-induced markers (E-cadherin, $\mathrm{N}$-cadherin, and Vimentin proteins), suggesting that targeted silencing of DLX6-AS1 impacts the invasive and metastatic phenotype of ESCC cells, partly by limiting the EMT process. Therefore, based on the results of previous studies and the results of the present study it was hypothesized that DLX6-AS1 probably functions as an oncogene while modulating ESCC pathogenesis.

The results of the present study confirmed the association between DLX6-AS1 expression, clinical characteristics of patients, and the proliferation and invasion of ESCC. Previous studies have stated that DLX6-AS1 expression promotes tumorigenesis and progression in hepatocellular carcinoma by regulating the miR-203a/matrix metalloproteinase- 2 pathway and renal cell carcinoma by modulating miR-26a/phosphatase and tensin homolog axis $(12,13)$. The present study was limited to fully elucidating the exact molecular mechanisms underlying DLX6-AS1-mediated gene regulation. Due to the diversity and complexity of the regulatory mechanisms of DLX6-AS1 in ESCC, further experiments are warranted to elucidate which signaling pathways are involved in the regulatory mechanism of DLX6-AS1 to expand the understanding of the effect of the high expression of DLX6-AS1 on the malignancy of ESCC in vitro and in vivo.

Overall, the present study demonstrated a higher prevalence of DLX6-AS1 overexpression in ESCC tissues when compared with the paired noncancerous tissues, suggesting that it could facilitate ESCC development and progression. Furthermore, the study also demonstrated that knockdown of DLX6-AS1 inhibited the growth and invasive abilities of the ESCC cell lines by promoting apoptosis and disrupting the EMT processes in vitro. Therefore, these results provide insight into the role of DLX6-AS1 as a potential therapeutic target in ESCC treatments. The application of DLX6-AS1 as a potential diagnostic and therapeutic target for ESCC progression and the determination of its precise mechanism of action should be further investigated in future studies.

\section{Acknowledgements}

Not applicable.

\section{Funding}

The present study was supported by a grant from the Support Plan of Science and Technology Innovation Team in Universities of Henan Province (grant no. 18IRTSTHN029).

\section{Availability of data and materials}

The datasets generated during the current study are available from the corresponding author on reasonable request.

\section{Authors' contributions}

SZ and MW conceived the study and participated in its design and coordination. YL performed the experiments and contributed to data collection. YY, XL and WY analyzed the data and drafted the manuscript. MZ assisted in designing experiments. YBL and KY provided technical expertise in conducting experiments. All authors read and approved the final manuscript. 


\section{Ethics approval and consent to participate}

Written informed consent was obtained from each patient prior to using the clinical samples for research purposes. The Ethics Committee of Zhengzhou University approved the study protocol (Henan, China).

\section{Patient consent for publication}

All patients agreed the publication of the research.

\section{Competing interests}

The authors declare that they have no competing interests.

\section{References}

1. Sugihara H, Ishimoto T, Miyake K, Izumi D, Baba Y, Yoshida N, Watanabe $\mathrm{M}$ and Baba $\mathrm{H}$ : Noncoding RNA expression aberration is associated with cancer progression and is a potential biomarker in esophageal squamous cell carcinoma. Int $\mathrm{J}$ Mol Sci 16: 27824-27834, 2015.

2. Torre LA, Bray F, Siegel RL, Ferlay J, Lortet-Tieulent J and Jemal A: Global cancer statistics, 2012. CA Cancer J Clin 65: 87-108, 2015.

3. Lin CY and Xu HM: Novel perspectives of long non-coding RNAs in esophageal carcinoma. Carcinogenesis 36: 1255-1262, 2015.

4. Holmes RS and Vaughan TL: Epidemiology and pathogenesis of esophageal cancer. Semin Radiat Oncol 17: 2-9, 2007.

5. Sjoquist KM, Burmeister BH, Smithers BM, Zalcberg JR, Simes RJ, Barbour A and Gebski V; Australasian Gastro-Intestinal Trials Group: Survival after neoadjuvant chemotherapy or chemoradiotherapy for resectable oesophageal carcinoma: An updated meta-analysis. Lancet Oncol 12: 681-692, 2011.

6. Ponting CP, Oliver PL and Reik W: Evolution and functions of long noncoding RNAs. Cell 136: 629-641, 2009.

7. Shen WJ, Zhang F, Zhao X and Xu J: LncRNAs and esophageal squamous cell carcinoma-implications for pathogenesis and drug development. J Cancer 7: 1258-1264, 2016.

8. Tsai MC, Manor O, Wan Y, Mosammaparast N, Wang JK, Lan F, Shi Y, Segal E and Chang HY: Long noncoding RNA as modular scaffold of histone modification complexes. Science 329: 689-693, 2010

9. Gutschner T and Diederichs S: The hallmarks of cancer: A long non-coding RNA point of view. RNA Biol 9: 703-719, 2012.

10. Huang C, Yuan N, Wu L, Wang X, Dai J, Song P, Li F, Xu C and Zhao X: An integrated analysis for long noncoding RNAs and microRNAs with the mediated competing endogenous RNA network in papillary renal cell carcinoma. Onco Targets Ther 10: 4037-4050, 2017.

11. Cui F, Wu D, He X, Wang W, Xi J and Wang M: Long noncoding RNA SPRY4-IT1 promotes esophageal squamous cell carcinoma cell proliferation, invasion, and epithelial-mesenchymal transition. Tumour Biol 37: 10871-10876, 2016.
12. Yao GL, Pan CF, Xu H, Wei K, Liu B, Zhai R and Chen YJ: Long noncoding RNA RP11-766N7.4 functions as a tumor suppressor by regulating epithelial-mesenchymal transition in esophageal squamous cell carcinoma. Biomed Pharmacother 88: 778-785, 2017.

13. Li J, Li P, Zhao W, Yang R, Chen S, Bai Y, Dun S, Chen X, Du Y, Wang Y, et al: Expression of long non-coding RNA DLX6-AS1 in lung adenocarcinoma. Cancer Cell Int 15: 48, 2015.

14. Zeng X, Hu Z, Ke X, Tang H, Wu B, Wei X and Liu Z: Long noncoding RNA DLX6-AS1 promotes renal cell carcinoma progression via miR-26a/PTEN axis. Cell Cycle 16: 2212-2219, 2017.

15. Zhang L, He X, Jin T, Gang L and Jin Z: Long non-coding RNA DLX6-AS1 aggravates hepatocellular carcinoma carcinogenesis by modulating miR-203a/MMP-2 pathway. Biomed Pharmacother 96: 884-891, 2017.

16. Edge SB and Compton CC: The American Joint Committee on Cancer: The 7th edition of the AJCC cancer staging manual and the future of TNM. Ann Surg Oncol 17: 1471-1474, 2010.

17. Livak KJ and Schmittgen TD: Analysis of relative gene expression data using real-time quantitative PCR and the 2(-Delta Delta C(T)) method. Methods 25: 402-408, 2001.

18. Rinn JL and Chang HY: Genome regulation by long noncoding RNAs. Annu Rev Biochem 81: 145-166, 2012.

19. Ge XS, Ma HJ, Zheng XH, Ruan HL, Liao XY, Xue WQ, Chen YB, Zhang Y and Jia WH: HOTAIR, a prognostic factor in esophageal squamous cell carcinoma, inhibits WIF-1 expression and activates Wnt pathway. Cancer Sci 104: 1675-1682, 2013.

20. Zhu W, Zhuang P, Song W, Duan S, Xu Q, Peng M and Zhou J: Knockdown of lncRNA HNF1A-AS1 inhibits oncogenic phenotypes in colorectal carcinoma. Mol Med Rep 16: 4694-4700, 2017.

21. Hauptman $\mathrm{N}$ and Glavac D: Long non-coding RNA in cancer. Int J Mol Sci 14: 4655-4669, 2013.

22. Chen X, Han H, Li Y, Zhang Q, Mo K and Chen S: Upregulation of long noncoding RNA HOTTIP promotes metastasis of esophageal squamous cell carcinoma via induction of EMT. Oncotarget 7: 84480-84485, 2016.

23. Deng HY, Wang YC, Ni PZ, Lin YD and Chen LQ: Long noncoding RNAs are novel potential prognostic biomarkers for esophageal squamous cell carcinoma: An overview. J Thorac Dis 8: E653-E659, 2016.

24. Tong YS, Wang XW, Zhou XL, Liu ZH, Yang TX, Shi WH, Xie HW, Lv J, Wu QQ and Cao XF: Identification of the long non-coding RNA POU3F3 in plasma as a novel biomarker for diagnosis of esophageal squamous cell carcinoma. Mol Cancer 14: 3, 2015.

25. Zheng X, Hu H and Li S: High expression of lncRNA PVT1 promotes invasion by inducing epithelial-to-mesenchymal transition in esophageal cancer. Oncol Lett 12: 2357-2362, 2016.

26. Yilmaz M and Christofori G: EMT, the cytoskeleton, and cancer cell invasion. Cancer Metastasis Rev 28: 15-33, 2009.

27. Huang C, Cao L, Qiu L, Dai X, Ma L, Zhou Y, Li H, Gao M, Li W, Zhang Q, et al: Upregulation of H19 promotes invasion and induces epithelial-to-mesenchymal transition in esophageal cancer. Oncol Lett 10: 291-296, 2015. 